\title{
Prevalence of Insomnia among Patients with the Ten Most Common Cancers in South Korea: Health Insurance Review and Assessment Service-National Patient Sample
}

\author{
Boram Park, MD, Soyoung Youn, MD, Chi-Won Christine Hann, BA, Kikyung Yi, MD, Suyeon Lee, MD, \\ Jung-Sun Lee, MD, PhD, Seockhoon Chung, MD, PhD \\ Department of Psychiatry, Asan Medical Center, University of Ulsan College of Medicine, Seoul, Korea
}

Background and Objective The insomnia rate in cancer patients is nearly three times higher than that in the general population. Due to the distinct and diverse nature of cancer, the prevalence of insomnia is assumed to be affected by differences in primary cancer sites. In this study, we explored the prevalence rates of insomnia among the ten most prevalent cancers in South Korea using a national patient sample.

Methods This was a 1-year cross-sectional study for the year 2011 using a national patient sample provided by the South Korean National Health Insurance. We selected all patients who had received International Classification of Disease, Tenth Revision (ICD-10) codes for the ten most prevalent cancers. To identify insomniacs, we included patients who had been diagnosed with ICD-10 codes or patients who had been prescribed with sleeping pills. The cancer and insomniac groups were subsequently merged and analyzed.

Results Insomnia was most prevalent in lung cancer (15.2\%), followed by non-Hodgkin's lymphoma (9.2\%), bladder (8.8\%), colorectal $(8.6 \%)$, stomach $(8.0 \%)$, prostate $(7.8 \%)$, breast $(7.8 \%)$, cervix $(7.8 \%)$, and liver $(6.6 \%)$ cancers, and was least prevalent in thyroid cancer patients $(5.8 \%)$. Within all cancer groups, insomniacs were significantly older than non-insomniacs. Insomnia predominance was not found in female cancer patients, which is in contrast to that typically seen in the general female population. In subgroup analysis, the prevalence of insomnia differed by both age and sex.

Conclusions The prevalence of insomnia varied according to cancer type. Patients with lung cancer were the most prone to insomnia. Since clinical and psychological factors may influence prevalence of insomnia, these factors will need closer study in the future.

Sleep Med Res 2016;7(2):48-54

Key Words Cancer, Insomnia, Psycho-oncology.

Received: June 21, 2016

Revised: August 9, 2016

Accepted: August 23, 2016

Correspondence

Seockhoon Chung, MD, PhD

Department of Psychiatry,

Asan Medical Center, University of Ulsan

College of Medicine, 88 Olympic-ro, 43 gil,

Songpa-gu, Seoul 05505, Korea

Tel +82-2-3010-3411

Fax +82-2-485-8381

E-mail_schung@amc.seoul.kr

\section{INTRODUCTION}

The insomnia rate in cancer patients is nearly three times higher than that in the general population. ${ }^{1}$ Several studies have revealed that $30-50 \%$ of cancer patients have sleep difficulties. $^{2}$ Sleep is often affected by cancer-related factors, including pain, distressing physical symptoms, long-term hospitalization, side effects from medication, and depression or anxiety. ${ }^{3,4}$ Cancer may also aggravate pre-existing insomnia. ${ }^{5}$ Moreover, sleep disturbance appears to increase the risk of cancer morbidity and related mortality. ${ }^{6}$ The prevalence of sleep disturbance in patients with cancer varies among studies because of many factors such as differences in the populations studied, cancer type, disease stage, the definition of sleep disturbance, and the mode of assessment.

In this study, we focused on differences in the prevalence of sleep disturbance according to cancer type. There are over 200 different types of cancer, each with its own symptoms, diagnostic and treatment methods, and prognosis. Given the distinct and diverse natures of different cancers, it is predicted that the prevalence of insomnia will vary with the primary cancer type. Previous insomnia research was conducted with relatively small samples and heterogeneous types of cancer, thereby limiting any comparison of the effects of different cancer types 
on insomnia. Several articles have reported significant differences in insomnia symptoms and its prevalence with respect to particular cancer types. For example, insomnia is reportedly more common in breast, lung, gastrointestinal, and gynecological cancer patients. However, these studies compared a limited number of randomly selected cancer types, and the study results are inconsistent. ${ }^{3,7}$

In South Korea, reliable clinical data determining the prevalence of insomnia among patients with different cancers is lacking. In this epidemiological study, we aimed to compare the prevalence rates of insomnia among the ten most frequent cancers occurring in the South Korean population using a national patient sample provided by the Korean National Health Insurance Service, i.e., across a large sample of cancer patients, and to analyze the impact of age and sex.

\section{METHODS}

We conducted a cross-sectional study using a national patient sample provided by the South Korean National Health Insurance for the year 2011. This study was approved by Institutional Review Board of Asan Medical Center.

\section{Korean Health Insurance Review and Assessment Service}

The Korean Health Insurance Review and Assessment Service-national patient sample (HIRA-NPS) consists of collected data from the national health insurance service of South Korea and presents anonymous information concerning diagnosis, medication, and hospital visits of its clients. ${ }^{8}$ The analyzed data set consisted of figures from 1375842 patients (3\% of total enrolled clients) who received medical treatment from January 2011 to December 2011. Data were extracted with random sampling methods and stratified by age and sex. The representativeness and validity of the sample were verified experimentally. ${ }^{9}$

The HIRA-NPS data contains a unique identification number for each patient, together with their age, sex, diagnosis, treatment method, treatment type (inpatient or outpatient), type of insurance, medical expenses, and prescriptions. Diagnosis was coded according to the International Classification of Disease, Tenth Revision (ICD-10), and prescriptions were recorded in generic drug names according to the Korean national code system.

\section{Study Subjects and Data Analysis}

Subjects under 18 years and over 100-years-old were excluded from the total of 1375842 patients, resulting in 1248914 patients being included for analysis in this study. Those coded with $\mathrm{C}$, corresponding to patients diagnosed with cancer, were singled out. ICD-10 codes that begin with the letter $\mathrm{C}$ are indicative of malignant neoplastic disorders. Among all $\mathrm{C}$ codes, we identified the ten most prevalent cancer types in Korea ${ }^{10}$ : thy- roid (C73), stomach (C16), colorectal (C18-C21), breast (C50), lung (C33 and C34), liver (C22), prostate (C61), cervix (C53), bladder (C67), and non-Hodgkin's lymphoma (NHL) (C82C85), in order of frequency. Patient demographics (age and sex) were merged and matched after the types of cancer were classified.

All patients diagnosed with a primary insomnia disorder (F51) or insomnia (G47.0) was selected initially. As insomnia can sometimes be episodic and does not necessitate a full psychiatric diagnosis, some individuals are prescribed sedative/ hypnotics without diagnosis. Hence, to take into account a broader range of individuals who needed medical help for insomnia symptoms, we included patients who had been prescribed with either zolpidem or triazolam. We then matched the patient lists of insomnia with the cancer patient lists to investigate the prevalence rates of insomnia.

Since this study examines the causal relationship between cancer type and development of insomnia, we extracted the dates of cancer diagnosis and insomnia diagnosis, or the first date of sedative/hypnotic prescription over the study period. By comparing the dates, patients who already had an insomnia diagnosis or sedative/hypnotics prescription prior to their first cancer diagnosis were excluded from the insomnia (due to cancer) group.

Patients with more than two cancer diagnoses before analysis were also excluded, to allow independent comparison between respective cancer types. All statistical processes stated above were conducted using STATA 13.1 version 1 (Stata Corp., College Station, TX, USA).

\section{Statistical Analysis}

The prevalence rate of insomnia is defined as the number of patients diagnosed with primary insomnia, or prescribed with classical sedative/hypnotics in each cancer type, divided by the total number of patients having each cancer type. The $\mathrm{p}$ value was calculated by Student's t-test for continuous variables (age) and chi-square test for categorical variables (sex). Tests were two-tailed, and $\mathrm{p}<0.05$ was considered statistically significant. Since cancers of individual organs generally have their own demographic distributions and characteristics, the relative contribution of age and sex to prevalence was examined by subgroup analyses. Subgroups were divided based on sex and age group at 20 year intervals (Group 1: 18-39 years, Group 2: 40-59 years, Group 3: 60-79 years, and Group 4: 80-100 years). Subgroup data were analyzed using chi-square test and Fisher's exact test. Pairwise comparisons were conducted to assess the differences in prevalence among cancer types, and the cancer having the highest prevalence in each subgroup was used as the reference. Significance levels for these comparisons were interpreted according to the Bonferroni correction, where a significance level of $\mathrm{p}<0.05 / 9=0.0056$ for each of the 9 comparisons was considered statistically significant. The above statistical analyses were 
carried out using SPSS version 21.0 (IBM Corp., Armonk, NY, USA).

\section{RESULTS}

\section{Study Sample Characteristics}

Among the 1248914 patients analyzed in this study, 33262 were diagnosed with one of the top ten cancer types. Of these, 2862 (8.6\%) patients with more than two cancers were excluded ( 2 sites: $\mathrm{n}=2585 ; 3$ sites: $\mathrm{n}=254 ; 4$ sites: $\mathrm{n}=21$; and 5 sites: $\mathrm{n}=$ 2 ), and the remaining 30400 (mean age, $58.1 \pm 13.8$ years; $48 \%$ male) comprised the final cancer sample analyzed. Thyroid cancer ( $\mathrm{n}=6249,0.50 \%$ ) was the most prevalent cancer type, followed by liver $(0.36 \%)$, colorectal $(0.34 \%)$, stomach $(0.34 \%)$, prostate $(0.26 \%)$, breast $(0.26 \%)$, lung $(0.19 \%)$, cervix $(0.09 \%)$, bladder $(0.07 \%)$ cancers, and NHL $(0.04 \%)$. The mean patient age was relatively higher for prostate $(66.3 \pm 11.2$ years $)$, lung $(65.1 \pm 13.0$ years $)$, and bladder $(65.0 \pm 13.2$ years $)$ cancers.
Male predominance was seen in bladder (73.1\%), liver (66.3\%), stomach $(64.3 \%)$, and lung $(62.7 \%)$ cancers. Female predominance was seen only in thyroid cancer ( $85.0 \%$ female). The general characteristics of the study sample are presented in Table 1.

\section{Prevalence of Insomnia}

The prevalence of insomnia was $8.21 \%$ (among 30400 patients). Insomnia was most prevalent in lung cancer (15.2\%), followed by NHL (9.2\%), bladder (8.8\%), colorectal (8.6\%), stomach $(8.0 \%)$, prostate $(7.8 \%)$, breast $(7.8 \%)$, cervix $(7.8 \%)$, and liver $(6.6 \%)$ cancer patients, and was least prevalent in thyroid cancer patients (5.8\%). Prevalence of insomnia in each type of cancer is displayed in Table 2 . The mean age was significantly higher for insomnia patients regardless of cancer type (Table 2). The age gap between insomnia and non-insomnia groups was highest in NHL (6.7 years) and lowest in lung cancer (2.6 years). Sex differences between insomnia and non-insomnia groups are shown in Fig. 1. Although female excess in prevalence of insomnia is generally well known, a predominance

Table 1. Prevalence of each cancer among all registered subjects aged 18-100

\begin{tabular}{|c|c|c|c|c|c|}
\hline & $\mathrm{n}$ & Prevalence (\%) & Male & Female & Mean age $\pm S D$ \\
\hline Thyroid & 6249 & 0.50 & $935(15.0 \%)$ & $5314(85.0 \%)$ & $50.2 \pm 12.1$ \\
\hline Liver & 4454 & 0.36 & $2951(66.3 \%)$ & $1503(33.7 \%)$ & $55.4 \pm 13.9$ \\
\hline Colorectal & 4274 & 0.34 & $2371(55.5 \%)$ & $1903(44.5 \%)$ & $62.4 \pm 12.6$ \\
\hline Stomach & 4222 & 0.34 & $2714(64.3 \%)$ & $1508(35.7 \%)$ & $62.1 \pm 12.3$ \\
\hline Prostate & 3288 & 0.26 & $3288(100.0 \%)$ & $0(0.0 \%)$ & $66.3 \pm 11.2$ \\
\hline Breast & 3120 & 0.26 & $16(0.5 \%)$ & 3104 (99.5\%) & $53.2 \pm 10.8$ \\
\hline Lung & 2332 & 0.19 & $1463(62.7 \%)$ & $869(37.3 \%)$ & $65.1 \pm 13.0$ \\
\hline Cervix & 1107 & 0.09 & $0(0 \%)$ & $1107(100 \%)$ & $53.0 \pm 13.2$ \\
\hline Bladder & 834 & 0.07 & $583(73.1 \%)$ & $251(26.9 \%)$ & $65.0 \pm 13.2$ \\
\hline NHL & 520 & 0.04 & $259(49.8 \%)$ & $259(49.8 \%)$ & $52.4 \pm 17.1$ \\
\hline Total & 30400 & 2.43 & $14580(48.0 \%)$ & $15820(52.0 \%)$ & $58.1 \pm 13.8$ \\
\hline
\end{tabular}

NHL: non-Hodgkin's lymphoma, SD: standard deviation.

Table 2. Prevalence of insomnia among each cancer and the mean age difference between insomnia and non-insomnia groups

\begin{tabular}{|c|c|c|c|c|c|c|c|}
\hline \multirow{2}{*}{ Cancer types } & \multirow{2}{*}{$\mathrm{n}$} & \multirow{2}{*}{$\begin{array}{l}\text { Prevalence } \\
\text { of insomnia }\end{array}$} & \multicolumn{2}{|c|}{ Insomnia group } & \multicolumn{2}{|c|}{ Non-insomnia group } & \multirow{2}{*}{ p-value } \\
\hline & & & $\mathrm{n}$ & Mean age $\pm S D$ & $\mathrm{n}$ & Mean age $\pm S D$ & \\
\hline Thyroid & 6249 & $5.8 \%$ & 363 & $55.8 \pm 12.0$ & 5886 & $49.8 \pm 12.1$ & $<0.001$ \\
\hline Colorectal & 4274 & $8.8 \%$ & 369 & $65.9 \pm 11.8$ & 3905 & $62.1 \pm 12.6$ & $<0.001$ \\
\hline Liver & 4454 & $6.6 \%$ & 295 & $60.7 \pm 12.7$ & 4159 & $55.0 \pm 13.9$ & $<0.001$ \\
\hline Stomach & 4222 & $8.0 \%$ & 338 & $65.7 \pm 12.0$ & 3884 & $61.8 \pm 12.3$ & $<0.001$ \\
\hline Prostate & 3288 & $7.8 \%$ & 256 & $70.5 \pm 10.6$ & 3032 & $65.9 \pm 11.1$ & $<0.001$ \\
\hline Breast & 3120 & $7.8 \%$ & 306 & $55.8 \pm 11.8$ & 2814 & $53.0 \pm 10.7$ & $<0.001$ \\
\hline Lung & 2332 & $15.2 \%$ & 354 & $67.3 \pm 10.8$ & 1978 & $64.7 \pm 12.4$ & $<0.001$ \\
\hline Cervix & 1107 & $7.8 \%$ & 81 & $58.5 \pm 14.5$ & 1026 & $52.6 \pm 13.0$ & $<0.001$ \\
\hline Bladder & 834 & $8.8 \%$ & 87 & $70.2 \pm 9.3$ & 747 & $64.4 \pm 13.4$ & $<0.001$ \\
\hline NHL & 520 & $9.2 \%$ & 48 & $58.5 \pm 13.3$ & 472 & $51.8 \pm 17.3$ & $<0.01$ \\
\hline
\end{tabular}

NHL: non-Hodgkin's lymphoma, SD: standard deviation. 


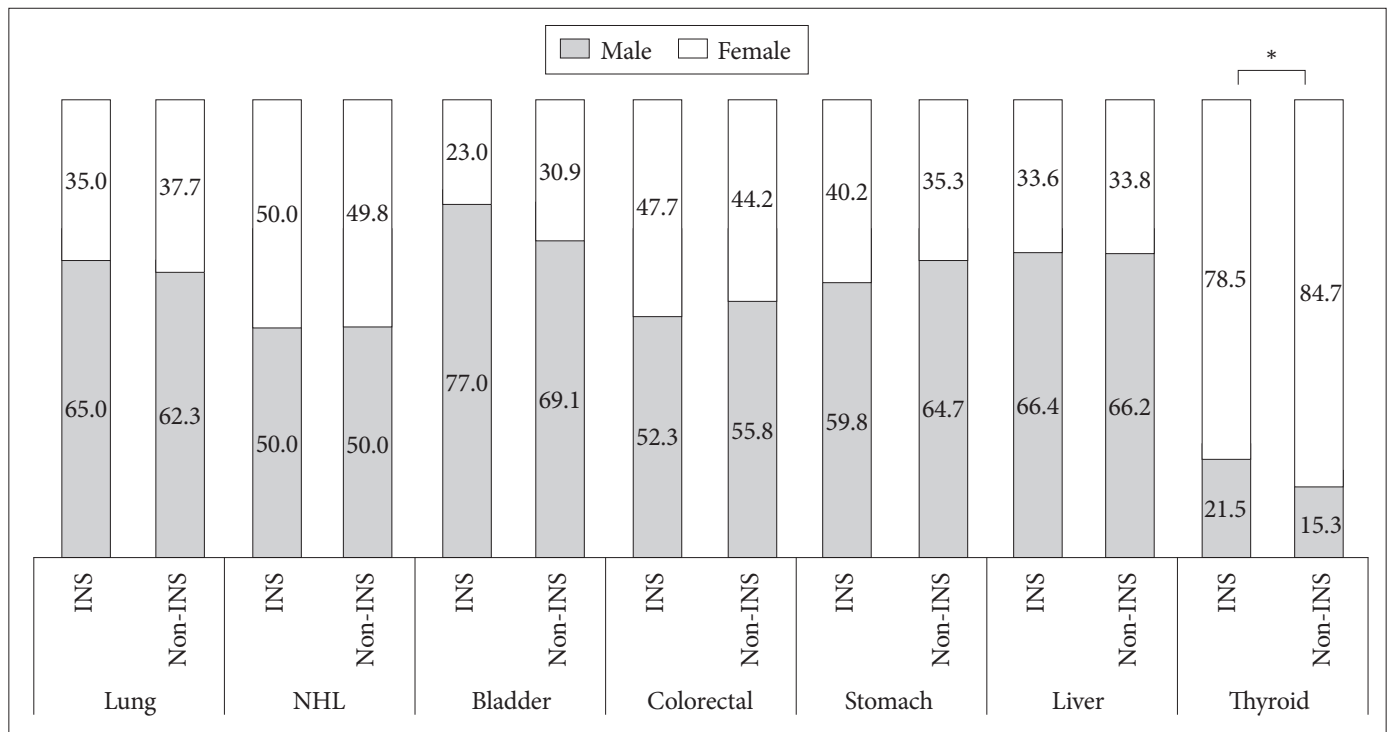

Fig. 1. Sex differences between insomnia and non-insomnia subgroups within each cancer type. Chi-square test: * $p<0.01$, sex difference is shown as a percentage (\%). INS: insomnia group, Non-INS: non-insomnia group.

Table 3. The prevalence of insomnia among each cancer subgroup

\begin{tabular}{ccrrrrrrrrrr}
\hline Sex & $\begin{array}{c}\text { Age group } \\
\text { (years) }\end{array}$ & Lung & Stomach & Colorectal & Liver & Bladder & NHL & Thyroid & Prostate & Cervix & Breast \\
\hline \multirow{2}{*}{ Male } & $18-39$ & $0.0 \%$ & $2.1 \%$ & $2.2 \%$ & $2.9 \%$ & $0.0 \%$ & $6.1 \%$ & $2.5 \%$ & $3.8 \%$ & - \\
& $40-59$ & $13.5 \%$ & $5.4 \%$ & $6.1 \%$ & $5.3 \%$ & $4.2 \%$ & $10.0 \%$ & $3.0 \%$ & $4.0 \%$ & - \\
& $60-79$ & $18.2 \%$ & $7.5 \%$ & $8.7 \%$ & $8.7 \%$ & $13.6 \%$ & $6.3 \%$ & $6.7 \%$ & $7.0 \%$ & - & - \\
& $80-100$ & $16.6 \%$ & $11.2 \%$ & $11.0 \%$ & $12.4 \%$ & $15.5 \%$ & $14.9 \%$ & $9.3 \%$ & $10.9 \%$ & - & - \\
& $18-1001$ & $15.7 \%$ & $7.4 \%$ & $8.1 \%$ & $6.6 \%$ & $11.5 \%$ & $9.2 \%$ & $3.7 \%$ & $7.8 \%$ & - & - \\
Female & $18-39$ & $4.4 \%$ & $7.0 \%$ & $4.5 \%$ & $4.2 \%$ & $0.0 \%$ & $3.4 \%$ & $2.5 \%$ & - & $6.0 \%$ & $7.2 \%$ \\
& $40-59$ & $12.5 \%$ & $6.9 \%$ & $7.0 \%$ & $5.3 \%$ & $6.7 \%$ & $9.6 \%$ & $6.0 \%$ & - & $4.8 \%$ & $8.5 \%$ \\
& $60-79$ & $16.4 \%$ & $9.2 \%$ & $9.1 \%$ & $6.5 \%$ & $10.0 \%$ & $13.3 \%$ & $9.3 \%$ & - & $10.5 \%$ & $13.1 \%$ \\
& $80-100$ & $15.4 \%$ & $11.4 \%$ & $12.5 \%$ & $11.0 \%$ & $9.1 \%$ & $11.1 \%$ & $12.3 \%$ & - & $16.1 \%$ & $16.2 \%$ \\
& $18-1001$ & $14.3 \%$ & $9.0 \%$ & $9.3 \%$ & $6.6 \%$ & $8.0 \%$ & $9.3 \%$ & $6.2 \%$ & - & $7.3 \%$ & $9.8 \%$ \\
\hline
\end{tabular}

NHL: non-Hodgkin's lymphoma.

of females in the insomnia group was not obvious in most cancer types. While colorectal and stomach cancers showed female predominance as expected, the sex ratio in liver cancer and NHL was relatively proportionate compared to non-insomnia groups. Although male predominance was found in the insomnia groups of lung, bladder, and thyroid cancers, a significant difference between sexes was only seen in thyroid cancer $(\mathrm{p}<0.01)$. Subgroup analysis reflected a tendency of increasing prevalence of insomnia with age in both sexes (Table 3). Overall comparisons of prevalence among cancer types were statistically significant $(\mathrm{p}<0.05)$ in the $40-59$ years, 60-79 years, and $80-100$ years subgroups of males and in the 18-39 years, 40-59 years, and 60-79 years subgroups of females (Table 4). Lung cancer showed the highest prevalence in the 40-59 years (13.5\% in male, $12.5 \%$ in female) and $60-79$ years $(18.2 \%$ in male, $16.4 \%$ in female) subgroups of both sexes and in the
80-100 years subgroup among males (16.6\%). Next, to lung cancer, insomnia in bladder cancer and NHL were also prevalent in male subgroups. In female groups, insomnia in breast cancer and NHL were also prevalent, following lung cancer. In the youngest female subgroup, prevalence of breast (7.2\%), stomach (7.0\%), and cervical (6.0\%) cancers was relatively higher than lung cancer (4.4\%). All alpha values for each pairwise comparison are shown in Table 4.

\section{DISCUSSION}

This cross-sectional study was conducted to explore differences in prevalence of insomnia between the ten most common cancer types in Korea. We used National Health Insurance claim data to compare the overall prevalence of insomnia in several 
Table 4. Pairwise comparison of each study subgroup

\begin{tabular}{|c|c|c|c|c|c|c|c|c|c|c|c|c|}
\hline \multirow{2}{*}{ Sex } & \multirow{2}{*}{$\begin{array}{l}\text { Age group } \\
\text { (years) }\end{array}$} & \multirow{2}{*}{$\begin{array}{c}\text { Overall } \\
\text { comparison }\end{array}$} & \multicolumn{10}{|c|}{ Pairwise comparison } \\
\hline & & & Lung & Stomach & Colorectal & Liver & Bladder & NHL & Thyroid & Prostate & Cervix & Breast \\
\hline \multirow[t]{4}{*}{ Male } & $18-39$ & 0.6406 & - & - & - & - & - & - & - & - & - & - \\
\hline & $40-59$ & $<0.001^{\ddagger}$ & Ref & $<0.0056^{\S}$ & $<0.0056^{\S}$ & $<0.0056^{\S}$ & $<0.0056^{\S}$ & 0.3481 & $<0.0056^{\S}$ & $<0.0056^{\S}$ & - & - \\
\hline & $60-79$ & $<0.001^{\ddagger}$ & Ref & $<0.0056^{\S}$ & $<0.0056^{\S}$ & $<0.0001$ & 0.1515 & 0.0417 & $<0.0056^{\S}$ & $<0.0056^{\S}$ & - & - \\
\hline & $80-100$ & $<0.05^{*}$ & Ref & 0.0051 & $<0.0056^{\S}$ & 0.0716 & 0.6886 & 0.7574 & 0.1581 & $<0.0056^{\S}$ & - & - \\
\hline \multirow[t]{4}{*}{ Female } & $18-39$ & $<0.05^{*}$ & 0.7526 & $>0.999$ & 0.3767 & 0.1777 & 0.6171 & 0.3948 & $<0.0056^{\S}$ & - & 0.598 & ref \\
\hline & $40-59$ & $<0.001^{\ddagger}$ & Ref & 0.0127 & 0.0099 & $<0.0056^{\S}$ & 0.1648 & 0.453 & $<0.0056^{\S}$ & - & 0.0072 & 0.0473 \\
\hline & $60-79$ & $<0.01^{\dagger}$ & Ref & 0.0085 & 0.0057 & $<0.0056^{\S}$ & 0.2242 & 0.5717 & $<0.0056^{\S}$ & - & 0.0781 & 0.2495 \\
\hline & $80-100$ & 0.3181 & - & - & - & - & - & - & - & - & - & - \\
\hline
\end{tabular}

$\mathrm{p}<0.05$ considered significant in overall comparisons. $\alpha<0.05 / 9=0.0056$ considered significant by Bonferroni correction in pairwise comparisons.

${ }^{*} \mathrm{p}<0.05 .{ }^{\dagger} \mathrm{p}<0.01 .{ }^{\ddagger} \mathrm{p}<0.001 .{ }^{\S_{\alpha}}<0.0056$.

Ref: reference, NHL: non-Hodgkin's lymphoma.

cancer types, and we also sought to assess psychiatric diagnosis and management in real clinical practice. Among the ten cancers assessed, insomnia was most prevalent in lung cancer patients (15.2\%). Cancer patients with insomnia showed a higher mean age across all cancer types. Although the higher prevalence of insomnia in females is generally well known, a predominance of females in the insomnia group in most cancer types was not obvious. In subgroup analysis, the prevalence of insomnia differed by both age and sex. Besides lung cancer, breast cancer showed the highest prevalence rate $(7.2 \%)$ in the subgroup containing the youngest females (18-39 years). In this study, prevalence of insomnia was $8.21 \%$ among the ten most common cancers. This rate of insomnia was higher than that reported in other Korean epidemiological studies. Lee et al. ${ }^{11}$ evaluated nonorganic sleep disorders (F51) among five major cancers (stomach, liver, colorectal, lung, and breast cancer) and found that $2.08 \%$ were diagnosed with sleep disorders. Kang, et al. ${ }^{12}$ also evaluated nonorganic sleep disorders (F51) among breast cancer patients, and the incidence of insomnia was $2.59 \%$. The higher prevalence of insomnia in our study may result from patients who took hypnotics without diagnosis. However, some designed studies showed a higher prevalence of insomnia than our result. Davidson, ${ }^{7}$ who evaluated cancer patients using Bergen insomnia scale, reported that $31 \%$ had clinical insomnia symptoms. Savard et al., ${ }^{5}$ who examined the prevalence of insomnia disorder in breast cancer patients using an insomnia screening questionnaire and interview, found that $20 \%$ met criteria for clinically significant insomnia. Taken together, it seems that insomnia is not only underdiagnosed in psychiatric clinics but under-recognized and under-treated even in medical clinics. The predominance of females among insomniacs has been shown in meta-analyses of large and qualified studies of the general population. ${ }^{13,14}$ In a Korean study, $22.8 \%$ of 5000 subjects of in the general population complained of insomnia with a prevalence significantly higher in women $(25.3 \%)$ than in men $(20.2 \%, \mathrm{p}<0.001) .{ }^{15}$ Surprisingly, we did not find a difference in sex distribution between insomnia and non-insomnia groups in this study. Sex differences in sleep quality and quantity are known to be influenced by physiological, psychological, and environmental factors. Women have a greater need for sleep, more frequent sleep arousal, greater symptom awareness, tendency to develop maladaptive sleep hygiene, and greater hormone variation than men. ${ }^{13}$ However, in cancer patients, we speculate that most insomnia is secondary insomnia, which is the result of tumor-related symptoms or side effects of treatment. Thus, sex disparity might not be as much as primary insomnia. Similar findings have been reported in a prospective study of cancer patients receiving chemotherapy. ${ }^{3}$ A recent study of breast cancer and prostate cancer also reported that cancer treatments and their side effects contribute to the aggravation of insomnia symptoms. ${ }^{16}$

In this study, the prevalence rate of insomnia was highest in lung cancer. Relatively higher prevalence of psychiatric comorbidity in lung cancer seems to be a consistent finding. Another Korean study using national registry data also identified a higher incidence of psychiatric disorders in lung cancer patients compared to other cancer patients. ${ }^{11}$ Similarly, a large crosssectional study showed that lung cancer patients had the highest prevalence rate ( $>50 \%$ ) of insomnia among cancer patients undergoing chemotherapy. ${ }^{3}$ A recent study of lung cancer also showed more than half the patients suffered from insomnia. In that study, pain medication, psychological distress, fatigue, pain, and chemotherapy itself contributed to sleep disturbance. ${ }^{17}$ It is known that lung cancer patients have relatively higher psychological and physical distress than other cancer patients. Zabora et al. ${ }^{18}$ compared the prevalence of psychological distress, such as depression, anxiety, hostility, somatization, interpersonal sensitivity, among a large sample of 14 cancer types ( $n=4496)$, and lung cancer showed the highest prevalence of distress (43.4\%). In addition, patients with lung cancer experience a variety of 
distressing physical symptoms, many of which continue throughout the course of the disease. Treatment-related symptoms also exacerbate disease-related symptoms and contribute to poor quality of life. Iyer et al. ${ }^{19,20}$ investigated symptom burden in advanced non-small cell lung cancer patients in Germany, France, and the USA. Fatigue, loss of appetite, shortness of breath, cough and pain were experienced consistently by a high proportion(> 90\%) of patients, and these symptoms, except for cough, were found to be significantly negative predictors of quality of life. Taken together, it seems that both psychological distress and a heavy symptom burden contribute to the high prevalence of insomnia.

NHL also showed a high prevalence of insomnia. NHL was found to be the fifth most distressful diagnosis among 14 cancer types. ${ }^{18}$ While insomnia in hematological cancers has not been greatly studied in adults, two studies have investigated factors worsening quality of life in hematological cancer. Both reported that insomnia was one of the most distressing symptoms, especially in relation to chemotherapy, with more than half of the patients reporting insomnia. ${ }^{21,22}$

Since epidemiological differences among cancer types can be a confounding factor in this study, we also analyzed age and sex subgroups. A relatively consistent tendency toward insomnia prevalence was seen in most age and sex subgroups, except in the youngest female subgroups. Thus, we concluded that the overall differences of prevalence rates are affected by differences of the disease nature and the therapeutic process of each cancer type. In most subgroups, lung cancer prevalence was highest, followed by NHL or bladder cancer. Thyroid cancer had a relatively lower prevalence rate than other types of cancer. However, we also found a subgroup-specific tendency of prevalence.

In subgroup analysis, female-specific cancers such as breast and cervical cancers showed a relatively high prevalence. The prevalence of insomnia in breast cancer was the highest (7.2\%) in the youngest age group (18-40 years). Insomnia was also more prevalent in breast cancer (16.2\%) and cervical cancer (16.1\%) in the oldest age group. In another cross-sectional study of insomnia, insomnia prevalence was highest in patients with breast and gynecological cancers. ${ }^{3}$ Insomnia has been reported problematic in young women with breast cancer, and over $50 \%$ of young patients reported insomnia after breast cancer diagnosis. ${ }^{19}$ Since a high prevalence of mood disorders such as depression and anxiety is well known in breast cancer, we assume that insomnia is be one of the symptoms of mood disorders. ${ }^{23,24}$ Another explanation is the relationship between insomnia and variation in sex hormones. Some women report increased insomnia at particular periods of the menstrual cycle, during pregnancy and the postpartum period, and during menopause. Similarly, changes in sex hormone levels lead to increased sleep disturbance. In addition, hormonal therapy, pelvic radiation, and removal of ovaries in breast and gynecological cancers can cause menopausal symptoms, including insomnia. ${ }^{25,26}$
To our knowledge, the present study is one of the few population-based studies describing psychiatric comorbidity in a sufficiently large population sample in South Korea. The majority of psychiatric studies in cancer patients are limited by small sample sizes, limited types of cancer, and different measurement tools. Thus, our large, randomly selected sample minimized selection bias, and made it possible to study several cancer types by the same approach. In addition, using insurance data, we were able to compare cancer and insomnia prevalence from a practical point of view involving psychiatric diagnosis by psycho-oncological services. Compared to other designed studies, the prevalence of insomnia tended to be relatively lower in our study probably due to under-diagnosis and undertreatment of insomnia in real clinical practice.

In consideration of current insurance systems in Korea, we screened $\mathrm{F}$ code, $\mathrm{G}$ code, and sedative/hypnotic prescription lists to include various insomnia types. Sedative/hypnotics are usually prescribed by various medical sectors for secondary insomnia. In these cases, patients usually take sedative/hypnotics without diagnosis or with G code diagnosis (G47.0). However, some patients suffer more severe and chronic insomnia requiring referral to a psychiatric department by medical doctors. If the insomnia is not a consequence of other psychiatric disorders, such as mood disorders or delirium, psychiatrists diagnose it as primary insomnia (F code, F51). Including various ways of identifying insomnia in (cancer) patients may increase the probability of reflecting the real insomnia burden in clinical practice.

This study had several limitations. First, cancer trajectory, illness duration, current stage, and previous history of psychiatric disorder were unmeasured confounding factors that could not be considered in this study. Second, the causal relationship between cancer and psychiatric conditions is not certain because of the study's cross-sectional design. Although we filtered patients who had insomnia diagnosed before the first cancer diagnosis (to add credence to a causal relationship), we could not completely overcome this limitation. Third, the reliability and validity of diagnosis is not as qualified as that in designed prospective longitudinal studies. Since diagnosis information in claims data ${ }^{27}$ is largely influenced by individual aspects of the patient and physician, as well as social, cultural, and economic backgrounds, we could not overlook the possibility of overand under-diagnoses. The study of the inaccuracy of diagnosis information of HIRA shows that diagnoses in the claims data tend to be more accurate in severe diseases, in hospitals and in inpatient settings rather than in mild diseases, in clinics, and in outpatient settings. Thus, further investigation into the operational definition of insomnia is needed to identify patients with insomnia who need significant clinical attention. Fourth, we did not include all sedative/hypnotics currently used in clinical practice. There are many benzodiazepines and non-benzodiazepines used for insomnia treatment, many of which are also used for other indications such as depression and anxiety. Our 
study included just two sedative/hypnotics-zolpidem and triazolam-that are used only for insomnia treatment. Thus, we assume that the true rate of insomnia in cancer may well be higher than our results suggest.

In conclusion, using National Health Insurance claim data for the ten most common cancers of South Korea, we found that the prevalence of insomnia was highest in lung cancer. Prevalence of insomnia in all cancer patients increases with age, as is also seen in the general population. However, female predominance was not significant among insomniac cancer patients. Males and females showed different patterns. Breast cancer and NHL showed relatively high numbers of females with insomnia, and bladder cancer and NHL showed relatively high numbers of males with insomnia. As clinical and psychological factors may influence prevalence of insomnia, such factors will need to be investigated more closely in future dedicated studies.

\section{Conflicts of Interest}

The authors have no financial conflicts of interest.

\section{REFERENCES}

1. Ohayon MM. Epidemiology of insomnia: what we know and what we still need to learn. Sleep Med Rev 2002;6:97-111.

2. Savard J, Morin CM. Insomnia in the context of cancer: a review of a neglected problem. J Clin Oncol 2001;19:895-908.

3. Palesh OG, Roscoe JA, Mustian KM, Roth T, Savard J, Ancoli-Israel S, et al. Prevalence, demographics, and psychological associations of sleep disruption in patients with cancer: University of Rochester Cancer Center-Community Clinical Oncology Program. J Clin Oncol 2010;28:292-8

4. Clark J, Cunningham M, McMillan S, Vena C, Parker K. Sleep-wake disturbances in people with cancer part II: evaluating the evidence for clinical decision making. Oncol Nurs Forum 2004;31:747-71.

5. Savard J, Simard S, Blanchet J, Ivers H, Morin CM. Prevalence, clinical characteristics, and risk factors for insomnia in the context of breast cancer. Sleep 2001;24:583-90.

6. Sigurdardottir LG, Valdimarsdottir UA, Fall K, Rider JR, Lockley SW, Schernhammer E, et al. Circadian disruption, sleep loss, and prostate cancer risk: a systematic review of epidemiologic studies. Cancer Epidemiol Biomarkers Prev 2012;21:1002-11.

7. Davidson JR, MacLean AW, Brundage MD, Schulze K. Sleep disturbance in cancer patients. Soc Sci Med 2002;54:1309-21.

8. Park YT, Yoon JS, Speedie SM, Yoon H, Lee J. Health insurance claim review using information technologies. Healthc Inform Res 2012;18:215-24.

9. Kim L, Sakong J, Kim Y, Kim S, Kim S, Tchoe B, et al. Developing the inpatient sample for the national health insurance claims data. Health Policy Manag 2013;23:152-61.

10. Jung KW, Won YJ, Kong HJ, Oh CM, Lee DH, Lee JS. Cancer statistics in Korea: incidence, mortality, survival, and prevalence in 2011. Can- cer Res Treat 2014;46:109-23.

11. Lee BO, Choi WJ, Sung NY, Lee SK, Lee CG, Kang JI. Incidence and risk factors for psychiatric comorbidity among people newly diagnosed with cancer based on Korean national registry data. Psychooncology 2015;24:1808-14.

12. Kang JI, Sung NY, Park SJ, Lee CG, Lee BO. The epidemiology of psychiatric disorders among women with breast cancer in South Korea: analysis of national registry data. Psychooncology 2014;23:35-9.

13. Zhang B, Wing YK. Sex differences in insomnia: a meta-analysis. Sleep 2006;29:85-93.

14. Chung S, Park B, Yi K, Lee J. Pattern of hypnotic drug prescription in South Korea: health insurance review and assessment service national patients sample. Sleep Med Res 2013;4: 51-5.

15. Cho YW, Shin WC, Yun CH, Hong SB, Kim J, Earley CJ. Epidemiology of insomnia in korean adults: prevalence and associated factors. J Clin Neurol 2009;5:20-3.

16. Savard J, Ivers H, Savard MH, Morin CM. Cancer treatments and their side effects are associated with aggravation of insomnia: results of a longitudinal study. Cancer 2015;121:1703-11.

17. Nishiura M, Tamura A, Nagai H, Matsushima E. Assessment of sleep disturbance in lung cancer patients: relationship between sleep disturbance and pain, fatigue, quality of life, and psychological distress. Palliat Support Care 2015;13:575-81.

18. Zabora J, BrintzenhofeSzoc K, Curbow B, Hooker C, Piantadosi S. The prevalence of psychological distress by cancer site. Psychooncology 2001;10:19-28.

19. Iyer S, Taylor-Stokes G, Roughley A. Symptom burden and quality of life in advanced non-small cell lung cancer patients in France and Germany. Lung Cancer 2013;81:288-93.

20. Iyer S, Roughley A, Rider A, Taylor-Stokes G. The symptom burden of non-small cell lung cancer in the USA: a real-world cross-sectional study. Support Care Cancer 2014;22:181-7.

21. Johnsen AT, Tholstrup D, Petersen MA, Pedersen L, Groenvold M. Health related quality of life in a nationally representative sample of haematological patients. Eur J Haematol 2009;83:139-48.

22. Andrade V, Sawada NO, Barichello E. [Quality of life in hematologic oncology patients undergoing chemotherapy]. Rev Esc Enferm USP 2013;47:355-61.

23. Gorman JR, Su HI, Roberts SC, Dominick SA, Malcarne VL. Experiencing reproductive concerns as a female cancer survivor is associated with depression. Cancer 2015;121:935-42.

24. Avis NE, Levine B, Naughton MJ, Case LD, Naftalis E, Van Zee KJ. Agerelated longitudinal changes in depressive symptoms following breast cancer diagnosis and treatment. Breast Cancer Res Treat 2013;139:199206.

25. Kravitz HM, Janssen I, Santoro N, Bromberger JT, Schocken M, Everson-Rose SA, et al. Relationship of day-to-day reproductive hormone levels to sleep in midlife women. Arch Intern Med 2005;165:2370-6.

26. Kravitz HM, Ganz PA, Bromberger J, Powell LH, Sutton-Tyrrell K, Meyer PM. Sleep difficulty in women at midlife: a community survey of sleep and the menopausal transition. Menopause 2003;10:19-28.

27. Park BJ, Sung J, Park K, Seo SW, Kim SH. Studying on diagnosis accuracy for health insurance claims data in Korea. Seoul: Seoul National University 2003;17-29. 